\title{
REVIEW
}

\section{Bone metabolism and fracture risk in type 2 diabetes mellitus}

\author{
Toru Yamaguchi and Toshitsugu Sugimoto
}

Department of Internal Medicine 1, Shimane University Faculty of Medicine, Shimane, Japan.

Osteoporosis and type 2 diabetes mellitus (T2DM), both prevalent in aging and westernized societies, adversely affect the health of elderly people by causing fractures and vascular complications, respectively. Recent experimental and clinical studies show that the disorders are etiologically related through the actions of osteocalcin and adiponectin. Meta-analyses of multiple clinical studies show that the hip fracture risk of T2DM patients is increased 1.4-1.7-fold compared with non-DM controls, even though the patients' bone mineral density (BMD) is not diminished. Vertebral fracture risk of the T2DM patients is also increased, and BMD measurement is not sensitive enough to assess this risk. These findings suggest that bone fragility in T2DM patients depends on bone quality deterioration rather than bone mass reduction. Surrogate markers are therefore needed to supplement the partial effectiveness of BMD testing in assessing the fracture risk of the T2DM patients. Markers related to advanced glycation end products may be candidates. These substances modulate bone quality in DM. Until research establishes the usefulness of surrogate markers, physicians should assess fracture risk in T2DM patients not only by measuring the BMD, but also by taking a fracture history and evaluating prior vertebral fractures using spinal $\mathrm{X}$-rays.

BoneKEy Reports 1, Article number: 36 (2012) | doi:10.1038/bonekey.2012.27

\section{Introduction}

The numbers of patients with osteoporosis or type 2 diabetes mellitus (T2DM) are increasing in aging and westernized societies. Both disorders predispose the elderly to disabling conditions and eventually raise mortality. Osteoporosis leads to fractures and T2DM leads to vascular complications. Although osteoporosis and T2DM have been viewed as separate diseases, accumulating evidence indicates that similar pathophysiological mechanisms underlie both of them.

\section{Interaction Between Bone Metabolism and Glucose and Fat Metabolism Through Osteocalcin and Wnt Signaling}

Osteocalcin (OC), a protein secreted by osteoblasts into the circulation, has several hormone-like features. ${ }^{1,2}$ Recent animal studies have shown that $\mathrm{OC}$ action is involved in the control of bone metabolism, as well as glucose metabolism and fat mass. ${ }^{3,4}$ In studies of OC-deficient mice, Lee et al. ${ }^{3}$ showed that OC functions as a hormone that improves glucose metabolism and reduces fat mass. Administration of recombinant uncarboxylated OC to wild-type mice fed a high-fat-diet-regulated gene expression in pancreatic $\beta$-cells and adipocytes (including adiponectin expression) and prevented development of metabolic diseases, obesity and hyperglycemia. ${ }^{4}$ The results of two recent experiments suggest the existence of a bone-pancreas endocrine loop in which bone resorption-dependent activation of OC occurs by way of the insulin receptor: ${ }^{5,6}$ insulin signaling in osteoblasts decreases osteoprotegerin expression in a Forkhead box O1-dependent manner. This promotes bone resorption and acidification of the bone extracellular matrix, which in turn promotes $\mathrm{OC}$ decarboxylation and, as a result, $\beta$-cell proliferation, insulin secretion and insulin sensitivity. Several clinical studies have also confirmed the relationship between OC, and glucose and fat metabolism in humans. ${ }^{7-11}$ We have shown previously that, in T2DM patients, the serum concentration of $\mathrm{OC}$ was negatively correlated with that of plasma glucose and with atherosclerosis parameters. ${ }^{7}$ We have also found that, in a population of patients evaluated for treatment of DM and osteoporosis, the serum concentration of undercarboxylated OC is correlated negatively with plasma glucose concentration and fat mass, and positively with serum adiponectin concentration. ${ }^{8}$ These experimental and clinical findings suggest that bone metabolism and glucose or fat metabolism are etiologically related through the action of

Correspondence: Dr T Yamaguchi, Department of Internal Medicine 1, Shimane University Faculty of Medicine, 89-1, Enya-cho, Izumo, Shimane 693-8501, Japan. E-mail: yamaguch@med.shimane-u.ac.jp 


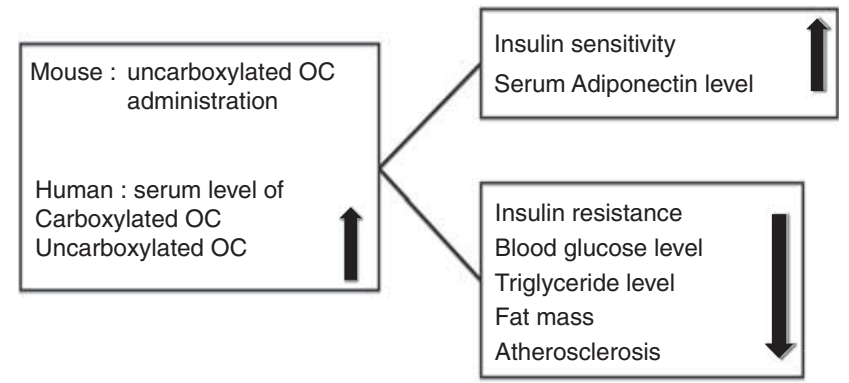

Figure 1 Relationship between osteocalcin $(\mathrm{OC})$ and various metabolic indicators in studies of mice and humans. Obese mice treated with uncarboxylated $\mathrm{OC}$ have increased blood insulin concentrations and decreased blood glucose, fat mass and triglycerides. In humans, serum carboxylated and uncarboxylated OC concentrations are correlated positively with insulin sensitivity and adiponectin concentration, and negatively with blood glucose, fat mass and atherosclerosis index. Thus, in both mice and humans, OC action pathophysiologically relates osteoporosis to diabetes.

OC (Figure 1). However, high glucose concentrations impair $\mathrm{OC}$ expression and secretion from the osteoblastic cells, ${ }^{12}$ and treatment of hyperglycemia in T2DM patients has increased serum OC concentrations in patients. ${ }^{13,14}$ Thus, in crosssectional studies in humans, the negative correlation between the concentration of serum $\mathrm{OC}$ and plasma glucose may be a result of hyperglycemia and not a cause for the suppression of insulin secretion and sensitivity.

Wnt signaling is also thought to be a common pathogenic pathway, important in osteoporosis and DM. A missense mutation in low-density lipoprotein receptor-related protein 6 , the co-receptor for the Wnt protein, has been genetically linked to osteoporosis, as well as DM, hyperlipidemia and coronary artery disease..$^{15}$ In addition, several studies have documented that $T$-cell-specific transcription factor- 4 , the partner of $\beta$-catenin in the canonical Wnt-signaling pathway, is the strongest T2DM susceptibility gene. ${ }^{16-19}$ Manolagas and Almeida, ${ }^{20}$ and other researchers ${ }^{21-23}$ have suggested that antagonism of Wnt signaling by oxidative stress diverts $\beta$-catenin from T-cell-specific transcription factor-mediated transcription to Forkhead box O-mediated transcription and contributes to the development of osteoporosis, as well as to insulin resistance and hyperlipidemia. In osteoblasts and their progenitors, inactivation of Forkhead box $\mathrm{O} 1$ by insulin signaling leads to decreased osteoblastogenesis by increasing reactive oxygen species abundance and oxidative stress, ${ }^{24}$ a mechanism implicated in the DM-related bone fragility, as discussed in a later section.

\section{Involvement of Fat Tissue in Bone Metabolism Through Adiponectin}

The positive correlation between bone mineral density (BMD) and fat mass, including the mass of visceral adipose tissue, suggests that there is a relationship between body fat and bone mass. ${ }^{25-27}$ However, the correlation between BMD and fat mass becomes negative when the mechanical loading effect of body weight on bone mass is considered, suggesting that body weight itself, but not fat mass, is related to increased BMD. ${ }^{28}$ Adiponectin is specifically and highly expressed in visceral, subcutaneous and bone marrow fat depots. It is also abundant in plasma and may have important roles in the regulation of energy homeostasis and insulin sensitivity. We and others have shown that osteoblasts have an adiponectin receptor and that the

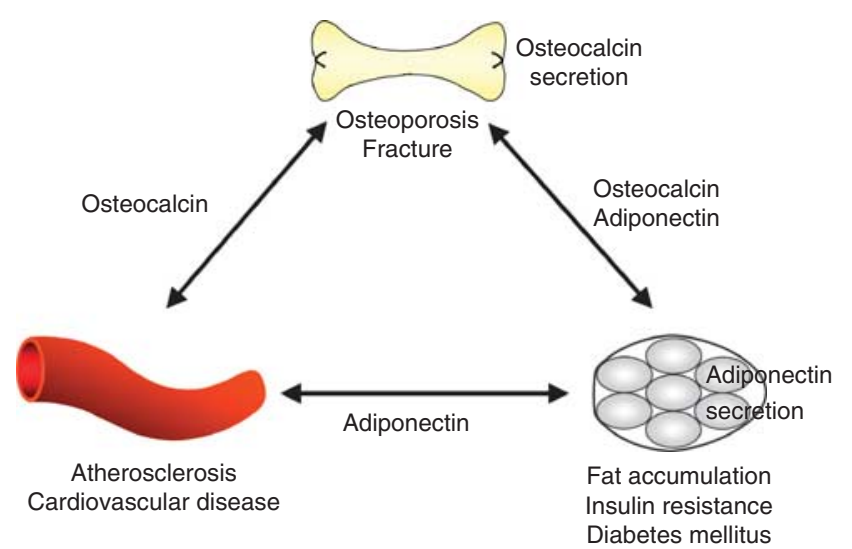

Figure 2 Interplay between bone metabolism and glucose or fat metabolism. Osteocalcin (OC) secreted from osteoblasts and adiponectin from fat cells may have beneficial effects on bone, fat tissue and arteries by protecting against metabolic derangement of these tissues.

proliferation, differentiation and mineralization of osteoblastic cells is enhanced by adiponectin. ${ }^{29,30}$ We have also found that serum adiponectin concentration is associated with BMD, bone turnover and the presence of vertebral fractures (VFs) in T2DM patients. ${ }^{31}$ In addition, we have found that, in poorly controlled T2DM patients, the serum adiponectin concentration before treatment predicts the subsequent increase in serum $\mathrm{OC}$ upon glycemic control. ${ }^{14}$ These findings suggest that adiponectin and $\mathrm{OC}$, secreted from fat tissue and osteoblasts, respectively, are involved in the interplay between the metabolisms of glucose or fat and bone (Figure 2).

\section{BMD and Fracture Risk in T2DM}

As a large proportion of the elderly population is affected by T2DM and osteoporosis, many clinical studies have investigated the association between the two. Although BMD is considered a gold standard for evaluating fracture risk in non-DM osteoporosis, accumulating evidence shows that T2DM patients have high fracture rates, despite the absence of decreased BMD. A recent meta-analysis showed that T2DM patients had higher hip BMD than non-DM controls $(z$-score $=0.27)$, despite an increased risk of hip fracture (1.4-fold) ${ }^{32}$ These data suggest that BMD values may not reflect bone fragility in T2DM. Another metaanalysis showed that hip fracture risk is increased 1.7-fold in T2DM patients. ${ }^{33}$ In contrast, recent data have indicated that the femoral neck BMD T- and fracture risk assessment-scores are associated with the risk of hip and non-spinal fractures among older adults with T2DM. ${ }^{34}$ These data suggest that BMD measurements are meaningful in T2DM. However, the same study has also shown that fracture risk was higher in T2DM subjects than in non-DM controls with a given T-score and age or with a given fracture risk assessment score. ${ }^{34}$ These findings suggest that femoral neck BMD and fracture risk assessment scores may be partially effective in assessing hip and non-spinal fracture risk in T2DM patients, although they may not be as sensitive as in non-DM subjects.

In contrast, little is known about the risk of VF and its association with BMD. To explore this association, we examined Japanese T2DM patients and non-DM controls. ${ }^{35,36}$ We found that, after adjustment for age, body mass index and lumbar BMD by logistic regression analysis, the presence of T2DM 
was an independent risk factor for prevalent radiographic VFs in women (odds ratio $(O R)=1.9)$, as well as men $(O R=4.7)$. Another logistic regression analysis showed that BMD at any site was not significantly associated with the presence of VFs in T2DM patients, in contrast to the significant association in non-DM controls. We examined the distribution of lumbar BMD as a function of age in non-DM and T2DM women (Figure 3). Non-DM subjects with VFs were clustered in the region of higher age and lower lumbar BMD. In contrast, there was no association with age or lumbar BMD for T2DM subjects with VFs. In addition, although quantitative ultrasound is considered useful in evaluating bone quality, we found that calcaneal quantitative ultrasound did not discriminate between T2DM patients with prevalent VFs and those without VFs. ${ }^{37}$ Thus, T2DM patients may have an increased risk of VFs that is independent of BMD or quantitative ultrasound values. These findings suggest that bone quality rather than bone mass may define bone fragility that causes hip fractures and VFs in T2DM.

\section{Surrogate Markers for Assessing Fracture Risk in T2DM}

As BMD is not sensitive enough to assess the risk of osteoporotic fractures in T2DM, it is important to explore the etiology of DM-related bone fragility to identify diagnostic markers that supplement the partial effectiveness of BMD.

Pentosidine. Advanced glycation end products (AGEs) are formed by the Maillard reaction, a collection of sequential nonenzymatic chemical glycoxidation reactions of protein amino groups. ${ }^{38}$ With normal aging, AGEs accumulate in various tissues, including the kidney and brain, and in coronary artery atherosclerotic plaques. However, hyperglycemia results in accelerated AGE formation, suggesting that AGEs have a pivotal role in the development of complications in DM patients. ${ }^{39,40}$ In addition, previous studies have revealed that AGEs accumulate in bone tissue, ${ }^{41,42}$ and the receptor for AGE (RAGE) is expressed in human bone-derived cells, ${ }^{43}$ suggesting that AGEs are associated with DM-related bone fragility.

Several studies have shown that AGEs have a negative impact on bone. AGEs inhibit the synthesis of type 1 collagen and $\mathrm{OC}$, as well as the formation of mature bone nodules in osteoblasts. ${ }^{44-46}$ We previously demonstrated that a combination of high glucose and AGE levels in vitro additively or synergistically inhibits the mineralization of osteoblastic cells through a glucose-induced increase in expression of RAGE. ${ }^{47}$ These findings suggest that accumulation of AGEs in bone may cause osteoblastic dysfunction. AGEs are also known to increase osteoclast activity. Previous in vitro and in vivo experiments ${ }^{48}$ have indicated that the number of resorption pits is increased when osteoclasts are cultured on AGE-modified dentin slices; moreover, AGE-modified bone particles implanted subcutaneously in rats are resorbed to a much greater extent than nonAGE-modified bone particles. In addition, RAGE knockout mice have fewer osteoclasts and a significantly higher bone mass than wild-type mice. ${ }^{49}$ Taken together, these findings indicate that AGE accumulation inhibits the differentiation and mineralization of osteoblasts and enhances the activity of osteoclasts, possibly leading to uncoupled bone turnover and resultant bone fragility.

Accumulation of AGE in bone is also negatively associated with the material properties of bone. ${ }^{41,42,50}$ Collagen crosslinks have critical roles in the determination of bone strength. ${ }^{51}$

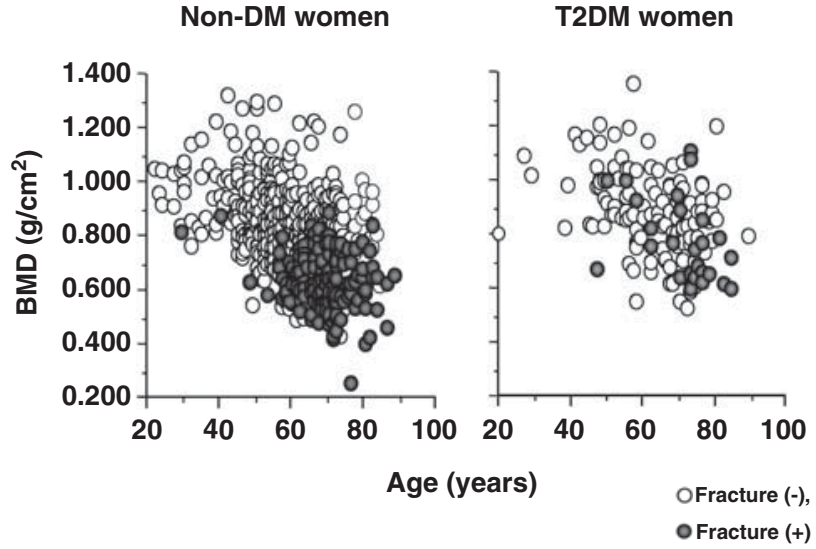

Figure 3 Lumbar bone mineral density (BMD) as a function of age in women without diabetes mellitus (DM) and those with type 2 diabetes mellitus (T2DM). Relative to the total population of non-DM women, non-DM subjects with vertebral fractures (VFs; closed circles) were clustered in the region of higher age and lower lumbar BMD. In contrast, T2DM subjects with VFs showed no clustering pattern. These findings suggest that BMD does not discriminate T2DM subjects with VFs from those without VFs (open circles).

AGE-type crosslinks, which are formed spontaneously by nonenzymatic glycation and oxidation reactions, are thought to be associated with the brittleness of collagen fibers, ${ }^{52,53}$ whereas physiological crosslinks (enzymatic crosslinks) strengthen collagen fibers and thus increase bone strength. ${ }^{42,54}$ For example, spontaneously diabetic WBN/Kob rats have decreased abundance of enzymatic crosslinks and increased abundance of AGE-type crosslinks; as a result, they show a deterioration in bone strength, despite the lack of BMD reduction. ${ }^{55}$

Pentosidine, one of the few AGEs characterized to date, is well known and chemically well defined. ${ }^{56-58}$ As the formation of pentosidine requires both glycation and oxidation, serum pentosidine concentration is considered a useful marker for glycoxidation. Several studies have revealed that pentosidine content in cortical or trabecular bone from vertebrae or femur is negatively associated with mechanical properties, ${ }^{41,42,50}$ and that the pentosidine content of cortical and trabecular bone derived from patients with femoral neck fracture is higher than that of age-matched controls. ${ }^{59,60}$ However, because invasive procedures such as bone biopsy are necessary for preparing specimens, assessment of bone pentosidine content is not easily done clinically. A recent study revealed that the plasma content of pentosidine is significantly and linearly correlated with that in the cortical bone. ${ }^{61}$ These data suggest that serum pentosidine concentration could be used as a surrogate marker for pentosidine content in bone and could indicate bone strength. We previously showed that serum pentosidine concentration was associated with the prevalence of VFs in postmenopausal T2DM women $\left(\mathrm{OR}=2.50\right.$ per s.d. increase) ${ }^{62}$ This association was independent of BMD, suggesting that it reflects bone quality rather than bone mass. In addition, an observational cohort study has shown that urine pentosidine concentration is associated with increased clinical fracture incidence in subjects with DM (relative hazard=1.42 per 1 s.d. increase in log pentosidine). ${ }^{63}$ Urinary pentosidine concentration at baseline also predicts future VFs in non-DM patients treated with bisphosphonate. ${ }^{64}$ Therefore, serum and urine pentosidine concentrations might be useful markers for assessing fracture risk in both T2DM and non-DM patients. 
Endogenous secretory RAGE. RAGE, a member of the immunoglobulin superfamily of cell surface receptors, interacts with multiple ligands, including AGEs. ${ }^{65}$ Crossbreeding of transgenic mice overexpressing human RAGE in their vascular cells with transgenic mice that develop insulin-dependent DM shortly after birth produced offspring that developed more progressive histological changes associated with DM nephropathy than did controls. ${ }^{66}$ This result confirmed that RAGE expression stimulates the development of DM complications. Endogenous secretory RAGE (esRAGE), a splice variant of one of the naturally occurring secretory forms of RAGE, contains the extracellular domains of the RAGE receptor, but lacks the transmembrane and cytoplasmic domains. ${ }^{67}$ Secreted esRAGE is thought to act as a decoy receptor that binds and neutralizes AGEs, reducing the activity of intercellular signal pathways that use RAGE. ${ }^{67}$ Indeed, administration of a genetically engineered soluble form of murine RAGE suppresses the development of diabetic atherosclerosis in a dose-dependent manner in streptozotocin-treated apoE-null DM mice. ${ }^{68}$ These findings suggest that enhanced RAGE activity is also linked to reduced bone strength in DM patients. Given the neutralizing activity of esRAGE, it is possible that the ratio of the serum concentrations of esRAGE and AGEs is more strongly linked to clinical bone problems, such as fractures, than is either parameter alone.

We found previously that the esRAGE/pentosidine ratio was significantly lower in T2DM patients with VFs than in patients without VFs. Multivariate logistic regression analysis, adjusted for age, height, weight, $\mathrm{HbA}_{1 \mathrm{c}}$, serum creatinine, DM duration, therapeutic agents, DM complications, osteoporotic risk factors and lumbar BMD, identified serum esRAGE concentration and esRAGE/pentosidine ratio as factors associated with the presence of VFs. The association was independent of BMD in men $(\mathrm{OR}=0.46$ and $\mathrm{OR}=0.34$ per s.d. increase, respectively) and in women $(\mathrm{OR}=0.32$ and $\mathrm{OR}=0.14$ per $\mathrm{s}$.d. increase, respectively). ${ }^{69}$ These results show that serum esRAGE concentration and the esRAGE/pentosidine ratio are more useful than BMD for assessing the risk of VFs in T2DM patients.

\section{Assessment and Prevention of Fracture Risk of T2DM Patients in Clinical Practice}

In the face of the partial effectiveness of BMD in assessing fracture risk in T2DM, the major clinical problems in preventing fractures in daily practice are how to assess the risk and when to start therapies. Although the markers related to AGEs are potential candidates for fracture-risk assessment, it is unclear whether they can be used to predict the occurrence of new fractures in T2DM patients.

A large study of the incidence of VFs in postmenopausal osteoporosis showed that, during a several-year study period, patients with VFs were more likely than those without, to suffer from new $V F s^{70,71}$ and hip fractures. ${ }^{72}$ This finding was independent of BMD. Thus, the presence of prevalent VFs might be useful for assessing bone quality in individual patients. Patient histories of existing non-VFs are also established risk factors for additional fractures. ${ }^{73}$ We found that 38 and $31 \%$ of men and women, respectively, with T2DM had prevalent VFs, as determined by X-ray. Sixteen percent of each group had histories of previous non-VFs. ${ }^{36}$ Thus, if patients with T2DM undergo spinal X-ray examination and are questioned about their fracture histories, it is likely that bone fragility will be identified in about half of the patients; those patients will need treatment for osteoporosis to prevent fractures. These procedures are simple, and we recommend that they be used by all physicians engaged in treating T2DM.

A recent study revealed that hip fracture-risk was higher, for a given T-score, in T2DM patients than in non-T2DM subjects. ${ }^{34}$ Therefore, when prevalent fractures are absent, osteopenic patients with T2DM and with BMD T-scores between -1.0 and -2.5 should undergo treatment to prevent fractures.

Large-scale studies of Caucasian and Chinese populations have shown that hip and other osteoporotic fracture risks are increased in patients with long-term or established T2DM. Fracture risks were not increased in those with impaired glucose tolerance or newly diagnosed T2DM. ${ }^{74-76}$ Thus, the severity and duration of T2DM should be considered when patients are started on drugs for osteoporosis.

It is unclear whether drugs for osteoporosis, such as bisphosphonates or selective estrogen-receptor modulators, are effective in preventing fractures in T2DM. However, recent studies have shown that the fracture-preventive potential of these drugs is similar in subjects with or without T2DM, ${ }^{77,78}$ suggesting that T2DM does not affect drug efficacy. Therefore, patients both with and without T2DM should receive the same treatments for osteoporosis.

\section{Summary}

T2DM populations are expanding in every country. It is therefore problematic that BMD testing is not sensitive enough to assess fracture risk - especially prevalent radiographic VF risk -in T2DM patients. If doctors use BMD values alone to diagnose osteoporosis, T2DM patients who are at risk of fracture may not be included in fracture-prevention programs. Practitioners should be aware of the importance of evaluating prior VFs and fracture histories by spinal X-ray and interview, as well as by measuring BMD. Combining all of these procedures would broaden the treatment of osteoporosis to include the T2DM population.

In addition, further studies are needed to clarify whether surrogate biochemical markers such as serum concentrations of pentosidine and esRAGE are useful for predicting the occurrence of new fractures in T2DM patients, and whether the sensitivity and specificity of these markers are similar to those of BMD measurement in osteoporotic non-DM patients.

\section{Conflict of Interest}

The authors declare no conflict of interest.

\section{References}

1. Hauschka PV, Lian JB, Cole DE, Gundberg CM. Osteocalcin and matrix protein: vitamin K-dependent proteins in bone. Physiol Rev 1989;69:990-1047.

2. Price PA. Gla-containing proteins of bone. Connect Tissue Res 1989;21:51-57.

3. Lee NK, Sowa H, Hinoi E, Ferron M, Ahn JD, Confavreux C et al. Endocrine regulation of energy metabolism by the skeleton. Cell 2007;130:456-469.

4. Ferron M, Hinoi E, Karsenty G, Ducy P. Osteocalcin differentially regulates beta cell and adipocyte gene expression and affects the development of metabolic diseases in wild-type mice. Proc Natl Acad Sci USA 2008;105:5266-5270.

5. Ferron M, Wei J, Yoshizawa T, Del Fattore A, DePinho RA, Teti A et al. Insulin signaling in osteoblasts integrates bone remodeling and energy metabolism. Cell 2010;142:296-308.

6. Fulzele K, Riddle RC, DiGirolamo DJ, Cao X, Wan C, Chen D et al. Insulin receptor signaling in osteoblasts regulates postnatal bone acquisition and body composition. Cell 2010;142 309-319. 
7. Kanazawa I, Yamaguchi T, Yamamoto M, Yamauchi M, Kurioka S, Yano S et al. Serum osteocalcin level is associated with glucose metabolism and atherosclerosis parameters in type 2 diabetes mellitus. J Clin Endocrinol Metab 2009:94:45-49.

8. Kanazawa I, Yamaguchi T, Yamamoto M, Yamauchi M, Kurioka S, Yano S et al. Serum undercarboxylated osteocalcin was inversely associated with plasma glucose level and fat mass in type 2 diabetes mellitus. Osteoporos Int. 2011;22:187-194.

9. Kindblom JM, Ohlsson C, Ljunggren O, Karlsson MK, Tivesten A, Smith U et al. Plasma osteocalcin is inversely related to fat mass and plasma glucose in elderly Swedish men. J Bone Miner Res 2009;24:785-791.

10. Fernandez-Real JM, Izquierdo M, Ortega F, Gorostiaga E, Gomez-Ambrosi J, Moreno-Navarrete $\mathrm{JM}$ et al. The relationship of serum osteocalcin concentration to insulin secretion, sensitivity, and disposal with hypocaloric diet and resistance training. J Clin Endocrinol Metab 2009;94: 237-245.

11. Pitass AG, Harris SS, Eliades M, Stark P, Dawson-Hughes B. Association between serum osteocalcin and markers of metabolic phenotype. J Clin Endocrinol Metab 2009;94:237-245.

12. Inaba M, Terada M, Koyama $\mathrm{H}$, Yoshida $\mathrm{O}$, Ishimura $\mathrm{E}$, Kawagishi $\mathrm{T}$ et al. Influence of high glucose on 1,25-dihydroxyvitamin D3-induced effect on human osteoblast-like MG-63 cells. J Bone Miner Res 1995;10:1050-1056.

13. Okazaki R, Totsuka Y, Hamano K, Ajima M, Miura M, Hirota Y et al. Metabolic improvement of poorly controlled noninsulin-dependent diabetes mellitus decreases bone turnover. J Clin Endocrinol Metab 1997:82:2915-2920.

14. Kanazawa I, Yamaguchi T, Yamamoto M, Yamauchi M, Yano S, Sugimoto T. Adiponectin is associated with changes in bone markers during glycemic control in type 2 diabetes mellitus. J Clin Endocrinol Metab 2009:94:3031-3037.

15. Mani A, Radhakrishnan J, Wang H, Mani A, Mani MA, Nelson-Williams $\mathrm{C}$ et al. LRP6 mutation in a family with early coronary disease and metabolic risk factors. Science 2007:315:1278-1282.

16. Smith U.TCF7L2 and type 2 diabetes-we WNT to know. Diabetologia 2007;50:5-7.

17. Grant SF, Thorleifsson G, Reynisdottir I, Benediktsson R, Manolescu A, Sainz J et al. Variant of transcription factor 7-like 2 (TCF7L2) gene confers risk of type 2 diabetes. Nat Genet 2006;38:320-323.

18. Owen KR, McCarthy MI. Genetics of type 2 diabetes. Curr Opin Genet Dev2007;17:239-244

19. Scott LJ, Mohlke KL, Bonnycastle LL, Willer CJ, LiY, Duren WL et al. A genome-wide association study of type 2 diabetes in Finns detects multiple susceptibility variants. Science 2007;316: 1341-1345.

20. Manolagas SC, Almeida M. Gone with the Wnts: $\beta$-catenin, T-cell factor, forkhead box 0 and oxidative stress in age-dependent diseases of bone, lipid, and glucose metabolism. Mol Endocrinol2007;21:2605-2614.

21. Hoogeboom D, Burgering BM. Should I stay or should I go: $\beta$-catenin decides under stress Biochim Biophys Acta 2009;1796:63-74.

22. DeCarolis NA, Wharton Jr KA, Eisch AJ. Which way does the Wnt blow? Exploring the duality of canonical Wnt signaling on cellular aging. Bioessays 2008;30:102-106

23. Jin T. The WNT signalling pathway and diabetes mellitus. Diabetologia 2008:51:1771-1780

24. Manolagas SC. From estrogen-centric to aging and oxidative stress: A revised perspective of the pathogenesis of osteoporosis. Endocr Rev2010;31:266-300.

25. Felson DT, Zhang Y, Hannan MT, Anderson JJ. Effects of weight and body mass index on bone mineral density in men and women. J Bone Miner Res 1993;8:567-573.

26. Glauber HS, Vollmer WM, Nevitt MC, Ensrud KE, Orwoll ES. Body weight versus body fat distribution, adiposity, and frame size as predictors of bone density. J Clin Endocrinol Metab 1995;80:1118-1123.

27. Yamaguchi T, Kanazawa I, Yamamoto M, Kurioka S, Yamauchi M, Yano S et al. Associations between components of the metabolic syndrome versus bone mineral density and vertebral fractures in patients with type 2 diabetes. Bone 2009:45:174-179.

28. Zhao LJ, Liu YJ, Liu PY, Hamilton J, Recker RR, Deng HW. Relationship of obesity with osteoporosis. J Clin Endocrinol Metab 2007;92:1640-1646.

29. Kanazawa I, Yamaguchi T, Yano S, Yamauchi M, Yamamoto M, Sugimoto T. Adiponectin and AMP kinase activator stimulate proliferation, differentiation, and mineralization of osteoblastic MC3T3-E1 cells. BMC Cell Biol 2007;8:51

30. Berner HS, Lyngstadaas SP, Spahr A, Monjo M, Thommesen L, Drevon CA et al. Adiponectin and its receptors are expressed in bone-forming cells. Bone 2004;35:842-849.

31. Kanazawa I, Yamaguchi T, Yamamoto M, Yamauchi M, Yano S, Sugimoto T. Relationships between serum adiponectin levels versus bone mineral density, bone metabolic markers, and vertebral fractures in type 2 diabetes mellitus. Eur J Endocrinol 2009;160:265-273.

32. Vestergaard P.Discrepancies in bone mineral density and fracture risk in patients with type 1 and type 2 diabetes-a meta-analysis. Osteoporos Int 2007;18:427-444.

33. Janghorbani M, Van Dam RM, WillettWC, Hu FB. Systematic review of type 1 and type 2 diabetes mellitus and risk of fracture. Am J Epidemiol 2007;166:495-505.

34. Schwartz AV, Vittinghoff E, Bauer DC, Hillier TA, Strotmeyer ES, Ensrud KE et al. Association of $\mathrm{BMD}$ and FRAX score with risk of fracture in older adults with type 2 diabetes. JAMA 2011;305:2184-2192.

35. Yamamoto M, Yamaguchi T, Yamauchi M, Kaji H, Sugimoto T. Bone mineral density is not sensitive enough to assess the risk of vertebral fractures in type 2 diabetic women. Calcif Tissue Int 2007;80:353-358

36. Yamamoto M, Yamaguchi T, Yamauchi M, Kaji H, Sugimoto T. Diabetic patients have an increased risk of vertebral fractures independent of bone mineral density or diabetic complications. J Bone Miner Res 2009:24:702-709.

37. Yamaguchi T, Yamamoto M, Kanazawa I, Yamauchi M, Yano S, Tanaka N et al. Quantitative ultrasound and vertebral fractures in patients with type 2 diabetes. J Bone Miner Metab 2011;29:626-632.
38. Brownlee M. Advanced protein glycosylation in diabetes and aging. Annu Rev Med 1995:46: 223-234

39. Brownlee M, Cerami A, Vlassara $\mathrm{H}$. Advanced glycosylation end products in tissue and the biochemical basis of diabetic complications. N Engl J Med 1988;318:1315-1321.

40. Goldin A, Beckman JA, Schmidt AM, Creager MA. Advanced glycation end products sparking the development of diabetic vascular injury. Circulation 2006;114:597-605.

41. Hernandez CJ, Tang SY, Baumbach BM, Hwu PB, Sakkee AN, van der Ham F et al. Trabecular microfracture and the influence of pyridinium and non-enzymatic glycation-mediated collagen cross-links. Bone 2005;37:825-832.

42. Wang $X$, Shen X, Li X, Agrawal CM. Age-related changes in the collagen network and toughness of bone. Bone 2002;31:1-7.

43. Takagi M, Kasayama S, Yamamoto T, Motomura T, Hashimoto K, Yamamoto H et al. Advanced glycation end-products stimulate interleukin- 6 production by human bone-derived cells. J Bone Miner Res 1997:12:439-446.

44. Yamamoto T, Ozono K, Miyauchi A, Kasayama S, Kojima Y, Shima M et al. Role of advanced glycation end products in adynamic bone disease in patients with diabetic nephropathy. $A m \mathrm{~J}$ Kidney Dis 2001;38 (Suppl 1): S161-S164.

45. Katayama Y, Akatsu T, Yamamoto M, Kugai N, Nagata N. Role of nonenzymatic glycosylation of type 1 collagen in diabetic osteopenia. J Bone Miner Res 1996;11:931-937.

46. Sanguineti R, Storace D, Monacelli F, Federici A, Odetti P. Pentosidine effects on human osteoblasts in vitro. Ann NY Acad Sci2008:1126:166-172.

47. Ogawa N, Yamaguchi T, Yano S, Yamauchi M, Yamamoto M, Sugimoto T. The combination of high glucose and advanced glycation end-products (AGEs) inhibits the mineralization of osteoblastic MC3T3-E1 cells through glucose-induced increase in the receptor for AGEs. Horm Metab Res 2007;39:871-875

48. Miyata T, Notoya K, Yoshida K, Horie K, Maeda K, Kurokawa K et al. Advanced glycation end products enhance osteoclast-induced bone resorption in cultured mouse unfractionated bone cells and in rats implanted subcutaneously with devitalized bone particles. J Am Soc Nephrol 1997;8:260-270.

49. Ding KH, Wang ZZ, Hamrick MW, Deng ZB, Zhou L, Kang B et al. Disordered osteoclast formation in RAGE-deficient mouse establishes an essential role for RAGE in diabetes related bone loss. Biochem Biophys Res Commun 2006:340:1091-1097.

50. Viguet-Carrin S, Roux JP, Arlot ME, Merabet Z, Leeming DJ, Byrjalsen I et al. Contribution of the advanced glycation end product pentosidine and of maturation of type I collagen to compressive biomechanical properties of human lumbar vertebrae. Bone 2006;39:1073-1079.

51. Saito M, Marumo K. Collagen cross-links as a determinant of bone quality: a possible explanation for bone fragility in aging, osteoporosis, and diabetes mellitus. Osteoporos Int 2010:21:195-214.

52. Garnero P, Borel O, Gineyts E, Duboeuf F, Solberg H, Bouxsein ML et al. Extracellular posttranslational modifications of collagen are major determinants of biomechanical properties of fetal bovine cortical bone. Bone 2006;38:300-309.

53. Vashishth D. The role of the collagen matrix in skeletal fragility. Curr Osteoporos Rep 2007;5 $62-66$

54. Banse X, Sims TJ, Bailey AJ. Mechanical properties of adult vertebral cancellous bone: correlation with collagen intermolecular cross-links. J Bone Miner Res 2002;17:1621-1628.

55. Saito M, Fujii K, MoriY, Marumo K. Role of collagen enzymatic and glycation induced cross-links as a determinant of bone quality in spontaneously diabetic WBN/Kob rats. Osteoporos Int 2006:17:1514-1523.

56. Odetti P, Fogarty J, Sell DR, Monnier VM. Chromatographic quantitation of plasma and erythrocyte pentosidine in diabetic and uremic subjects. Diabetes 1992;41:153-159.

57. Monnier VM, Sell DR, Nagaraj RH, Miyata S, Grandhee S, Odetti P et al. Maillard reactionmediated molecular damage to extracellular matrix and other tissue proteins in diabetes, aging, and uremia. Diabetes 1992;41 (Suppl 2):36-41.

58. Grandhee SK, Monnier VM. Mechanism of formation of the Maillard protein cross-link pentosidine. Glucose, fructose, and ascorbate as pentosidine precursors. J Biol Chem 1991;266:11649-11653.

59. Saito M, Fujii K, Soshi S, Tanaka T. Reductions in degree of mineralization and enzymatic collagen cross-links and increases in glycation-induced pentosidine in the femoral neck cortex in cases of femoral neck fracture. Osteoporos Int 2006;17:986-995.

60. Saito M, Fujii K, Marumo K. Degree of mineralization-related collagen crosslinking in the femora neck cancellous bone in cases of hip fracture and controls. Calcif Tissue Int 2006:79:160-168.

61. Odetti P, Rossi S, Monacelli F, Poggi A, Cirnigliaro M, Federici M et al. Advanced glycation end products and bone loss during aging. Ann NY Acad Sci2005;1043:710-717.

62. Yamamoto M, Yamaguchi T, Yamauchi M, Yano S, Sugimoto T. Serum pentosidine levels are positively associated with the presence of vertebral fractures in postmenopausal women with type 2 diabetes. J Clin Endocrinol Metab 2008:93:1013-1019.

63. Schwartz AV, Garnero P, Hillier TA, Sellmeyer DE, Strotmeyer ES, Feingold KR et al. Pentosidine and increased fracture risk in older adults with type 2 diabetes. $J$ Clin Endocrinol Metab 2009:94:2380-2386.

64. Shiraki M, Kuroda T, Shiraki Y, Tanaka S, Higuchi T, Saito M. Urinary pentosidine and plasma homocysteine levels at baseline predict future fractures in osteoporosis patients under bisphosphonate treatment. J Bone Miner Metab 2011;29:62-70.

65. Kislinger T, Fu C, Huber B, Qu W, Taguchi A, Du Yan S et al. N(epsilon)-(carboxymethyl)lysine adducts of proteins are ligands for receptor for advanced glycation end products that activate cell signaling pathways and modulate gene expression. J Biol Chem 1999;274:31740-31749.

66. Yamamoto Y, Kato I, Doi T,Yonekura H, Ohashi S, Takeuchi M et al. Development and prevention of advanced diabetic nephropathy in RAGE-overexpressing mice. J Clin Invest 2001;108:261-268.

67. Yonekura H, Yamamoto Y, Sakurai S, Petrova RG, Abedin MJ, Li H et al. Novel splice variants of the receptor for advanced glycation end-products expressed in human vascular endothelial cells 
and pericytes, and their putative roles in diabetes-induced vascular injury. Biochem J 2003:370 (Part 3): 1097-1109.

68. Park L, Raman KG, Lee KJ, Lu Y, Ferran Jr LJ, Chow WS et al. Suppression of accelerated diabetic atherosclerosis by the soluble receptor for advanced glycation endproducts. Nat Med 1998;4:1025-1031.

69. Yamamoto M, Yamaguchi T, Yamauchi M, Sugimoto T. Low serum level of the endogenous secretory receptor for advanced glycation end-products (esRAGE) is a risk factor for prevalent vertebral fractures independent of bone mineral density in patients with type 2 diabetes. Diabetes Care 2009;32:2263-2268.

70. Black DM, Arden NK, Palermo L, Pearson J, Cummings SR. Prevalent vertebral deformities predict hip fractures and new vertebral deformities but not wrist fractures. Study of Osteoporotic Fractures Research Group. J Bone Miner Res 1999;14:821-828.

71. Liberman UA, Weiss SR, Broll J, Minne HW, Quan H, Bell NH et al. Effect of oral adendronate on bone mineral density and the incidence of fractures in postmenopausal osteoporosis. The Alendronate Phase III Osteoporosis Treatment Study Group. N Engl J Med 1995;333 1437-1443.
72. Kanis JA, Borgstrom F, De Laet C, Johansson H, Johnell O, Jonsson B et al. Assessment of fracture risk. Osteoporosis Int 2005;16:581-589.

73. Kanis JA, Oden A, Johansson H, Borgström F, Ström O, McCloskey E. FRAX and its applications to clinical practice. Bone $2009 ; 44: 734-743$.

74. de Liefde II, van der Klift M, de Laet CE, van Daele PL, Hofman A, Pols HA. Bone mineral density and fracture risk in type-2 diabetes mellitus: the Rotterdam Study. Osteoporos Int 2005;16:1713-1720.

75. Leslie WD, Lix LM, Prior HJ, Derksen S, Metge C, O’Neil J. Biphasic fracture risk in diabetes: a population-based study. Bone 2007;40:1595-1601.

76. Koh WP, Wang R, Ang LW, Heng D, Yuan JM, Yu MC. Diabetes and risk of hip fracture in the Singapore Chinese Health Study. Diabetes Care 2010;33:1766-1770.

77. Ensrud KE, Stock JL, Barrett-Connor E, Grady D, Mosca L, Khaw KT et al. Effects of raloxifene on fracture risk in postmenopausal women: the raloxifene use for the heart trial. $J$ Bone Miner Res 2008;23:112-120.

78. Vestergaard $P$, Rejnmark $L$, Mosekilde $L$. Are antiresorptive drugs effective against fractures in patients with diabetes? Calcif Tissue Int 2011;88:209-214. 